\title{
Physician judgement is a crucial adjunct to pneumonia severity scores in low-risk patients
}

\author{
G. Choudhury*, J.D. Chalmers*, P. Mandal*, A.R. Akram*, M.P. Murray*, P. Short*, \\ A. Singanayagam* and A.T. Hill*
}

ABSTRACT: This study investigates the reasons for hospitalisation in patients with low-risk (CURB-65 score 0-1) community-acquired pneumonia (CAP), with a view to identifying the potential for improving outpatient management.

As part of a prospective observational study of CAP, we evaluated reasons for hospitalisation in these low-risk patients.

565 patients had low-risk CAP and 420 of these were admitted (for $>12 \mathrm{~h}$ ). $39.3 \%$ had additional markers of severity justifying admission, $29.5 \%$ of the admissions were required for further management that could not be provided rapidly in the community, $11.9 \%$ had unsafe social circumstances and $19.3 \%$ had no clinical reason justifying hospitalisation. 30-day mortality was increased in patients with additional severity markers (6.7\%), which was significantly higher compared with $0 \%$ for patients awaiting investigations $(p=0.009)$ and $0 \%$ without a clear indication for hospitalisation $(p=0.04)$. In a logistic regression analysis, parameters associated with 30 -day mortality were chronic cardiac comorbidity (adjusted odds ratio (aOR) 5.73, 95\% Cl 1.52-21.6; $\mathrm{p}=\mathbf{0 . 0 1}$ ), acidosis (aOR 5.14, 95\% Cl 1.44-18.3; $\mathrm{p}=0.01$ ), hypoxia (aOR 9.86, 95\% Cl 2.39-40.7; $\mathrm{p}=0.002)$ and multilobar chest radiograph shadowing (aOR 4.54, 95\% $\mathrm{Cl} 1.21-17.1 ; \mathrm{p}=0.03)$.

This study supports recommendations from international guidelines that pneumonia severity scores should be used as an adjunct to clinical judgement, when deciding on hospitalisation.

KEYWORDS: Lung, infection, infectious diseases pneumonia

ommunity-acquired pneumonia (CAP), the most common infectious disease requiring hospitalisation in western countries, accounts for $\sim 100,000$ hospital admissions per year in England [1]. Admissions for pneumonia are rising, particularly in elderly patients, and this is a major burden on healthcare resources [2].

In 1997, GUEST and MORRIS [3] showed that patients hospitalised with CAP represent only $32 \%$ of the total burden of pneumonia but $96 \%$ of the annual costs, with an average cost of $£ 1,700-£ 5,100$ per patient per hospital admission compared with $£ 100$ per episode in the community. Therefore, the cost of in-patient care is much higher than outpatient care and accounts for the majority of the resources used annually, irrespective of pneumonia severity.

CAP was estimated to cost the National Health Service (NHS) $\sim £ 440.7$ million per year in the 1992-1993 healthcare assessment [3]. Intervention studies consistently show that by applying objective criteria for admission, many inappropriate hospitalisations can be avoided [4]. Inappropriate admissions contribute to problems such as Clostridium difficile and methicillin-resistant Staphylococcus aureus infection, in addition to increasing hospital costs [5]. Due to these costs and the possible hazards, it is important to admit only those who will benefit from in-patient care.

The Infectious Disease Society of America, American Thoracic Society and British Thoracic Society (BTS) recommend the use of prognostic scoring tools, such as the Pneumonia Severity Index (PSI) and CURB-65 score (confusion, urea $>7 \mathrm{mmol} \cdot \mathrm{L}^{-1}$, respiratory frequency $\geqslant 30$ breaths. $\min ^{-1}$, systolic blood pressure $<90 \mathrm{mmHg}$ or diastolic blood pressure $\leqslant 60 \mathrm{mmHg}$, and age $\geqslant 65 \mathrm{yrs})$, for severity assessment for hospital admission [6-10]. The PSI is the most widely used severity assessment tool and is composed of 20 patient variables, including demographics, comorbidities, and clinical, laboratory and radiological variables [6]. CURB-65 is an alternative severity score proposed by the BTS $[7,11]$. It is significantly simpler to calculate, being composed of only five
AFFILIATIONS

*Dept of Respiratory Medicine, Royal Infirmary of Edinburgh, Edinburgh, and

"Dept of Respiratory Medicine, Ninewells Hospital and Medical School, Dundee, UK.

CORRESPONDENCE

G. Choudhury

Dept of Respiratory Medicine Royal Infirmary of Edinburgh

51 Little France Crescent

Edinburgh

EH16 4SA

UK

E-mail: gourab19@hotmail.com

Received:

Nov 052010

Accepted after revision:

Feb 192011

First published online:

March 152011 
variables, and has been shown to perform similarly to the PSI in predicting 30-day mortality [12-15]. The BTS CAP guidelines recommend that patients in low-risk categories using the CURB65 score (0-1) are at low risk of mortality and, therefore, can be managed as outpatients [7].

Despite the availability of these scores and the finding that outpatient management for selected patients is both safe and acceptable to patients, studies continue to show that the majority of low-risk patients presenting to hospital are admitted [4, 16-23].

The reasons for this and the potential for increasing outpatient management using the CURB-65 score have not been extensively studied.

The aim of this study was to investigate the reasons for hospital admission in patients with low CURB-65 scores (0-1) and identify the potential for improving outpatient management of low-risk patients with CAP.

\section{METHODS}

We retrospectively reviewed case records for CAP patients admitted with low CURB-65 scores (0-1) enrolled in a prospective observational study of CAP conducted from 2005 to 2008. CURB-65 was part of the hospitalisation protocol for patients admitted to the study hospitals. The study was conducted in NHS Lothian, Edinburgh, UK. Patients were considered for inclusion in the study if they had a primary diagnosis of CAP.

\section{Inclusion and exclusion criteria}

Patients were included in the study if they presented with a new infiltrate on a chest radiography and had three or more symptoms or signs consistent with pneumonia (new or increased shortness of breath, cough, sputum production, sputum purulence, haemoptysis, chest pain, fever/rigors, or signs consistent with pneumonia on chest auscultation), along with a CURB-65 score of 0-1 (1 point was given for each of the following on admission: new onset confusion (abbreviated mental test score $\leqslant 8$ ), raised urea $>7 \mathrm{mmol} \cdot \mathrm{L}^{-1}$, respiratory rate $\geqslant 30$ breaths $\cdot \mathrm{min}^{-1}$, systolic blood pressure $<90 \mathrm{mmHg}$ and/or diastolic blood pressure $\leqslant 60 \mathrm{mmHg}$, and age $\geqslant 65 \mathrm{yrs}$ ).

Exclusion criteria included: hospital-acquired pneumonia (development of symptoms $>48 \mathrm{~h}$ following admission or discharge from an acute care facility $<2$ weeks prior to admission); age <18 yrs; active thoracic malignancy; immunosuppression (including maintenance corticosteroid therapy at any dose); pulmonary embolism; active tuberculosis; and patients in whom active treatment was not considered appropriate at the time of admission (palliative care).

\section{Retrospective records review}

We reviewed all cases of CAP with a CURB-65 score of 0-1 enrolled in the observational study. Two investigators reviewed each case independently. In the case of a disagreement between reviewers, a third independent reviewer had the casting vote. The reviewers were not involved in the initial care of these patients. Reviewers were blinded to patient outcome at the time of review and were only provided with information available at the time of the initial admission decision. The investigators were asked to determine whether, if provided with the available information, they would have hospitalised the patient or considered them for outpatient care. Where reviewers felt the patient should be hospitalised they were asked to provide a reason. Once this process was complete, patients were classified into the following categories: 1) patients who were discharged from hospital (for the purposes of this study, any patient spending $<12 \mathrm{~h}$ in the emergency department or medical admissions unit before being discharged was regarded as discharged from hospital); 2) those who were admitted because of clinician concern, where additional markers of severity were identified that may increase the risk of mortality and, therefore, necessitate inpatient care; 3) patients hospitalised without any additional markers of severity, but requiring additional investigations (for example, to exclude an alternative diagnosis or investigate abnormal results identified on admission) where such investigations could not be provided quickly as an outpatient; 4) those admitted because unsafe or inadequate social circumstances made discharge inappropriate (unmet social needs); and 5) patients where no clinical reason for hospitalisation could be identified.

\section{Outcomes}

The aim of the study was to determine reasons for hospitalisation in low-risk patients with CAP. We assessed 30-day mortality for all patients in the study. Follow-up was conducted by outpatient clinic review or by phone call to the patients' general practitioner in patients not attending for outpatient review. Survival status was confirmed in $100 \%$ of patients.

\section{Statistical analysis}

All data were analysed using SPSS version 13 for Windows (SPSS Inc., Chicago, IL, USA). Descriptive statistics of demographic and clinical variables are presented as median (interquartile range), unless otherwise stated. The Chi-squared test was used to compare categorical data between groups, with Fisher's exact test used where any cell contained $<10$. The Mann-Whitney U-test was used for comparison of two groups of continuous data. Multivariable logistic regression was used to identify markers of severity associated with 30-day mortality in this low-risk group. The aim was to identify markers additional to the CURB-65 criteria to predict mortality in this group. All clinical, laboratory and demographic variables were included in a logistic regression model. All patients had mild CURB-65 score (0-1). Model adequacy was assessed using the Hosner-Lemeshow goodness-of-fit test. For all analyses, a p-value $<0.05$ was considered statistically significant.

\section{RESULTS}

The authors studied 1,472 patients with CAP over a 3-yr period. After exclusion of patients with CURB-65 scores 2-5, 565 patients were classified into CURB-65 score 0-1. 145 patients were discharged within $12 \mathrm{~h}$ of admission to hospital and 420 patients were admitted to hospital with mild CAP (CURB-65 score 0-1).

The demographic characteristics of the study groups are shown in table 1 . There was a greater proportion of patients with chronic obstructive pulmonary disease (COPD) and chronic cardiac disease (COPD 21.2\% versus $9.0 \%, \mathrm{p}=0.01$, 
and chronic cardiac disease $13.1 \%$ versus $4.8 \%, \mathrm{p}=0.02$ ) in those admitted to the hospital for $>12 \mathrm{~h}$.

Table 2 compares parameters of CURB-65 between the two groups. The only significant difference was that patients admitted to the hospital for $>12 \mathrm{~h}$ had a respiratory rate of $\geqslant 30$ breaths $\cdot \mathrm{min}^{-1}$. Small numbers of patients were discharged having had abnormal values for respiratory rate and systolic blood pressure on admission (table 2). In the majority of cases, these values improved with initial treatment (such as fluid management, nebuliser, analgesia and reassurance). Three out of the five patients with a respiratory rate of $\geqslant 30$ breaths $\cdot \mathrm{min}^{-1}$ on admission had a diagnosis of COPD and received initial bronchodilator treatment. Of those patients with low systolic blood pressure $<90 \mathrm{mmHg}$, two were young female patients with no other markers of systemic illness and one further patient's blood pressure improved over a few hours with initial treatment.

Despite similar CURB-65 scores, hospitalised patients often had multiple additional markers of severity. Table 3 compares other clinical and investigative parameters in both the groups. Patients admitted to the hospital for $>12 \mathrm{~h}$ had an increased frequency of hypo- or hyperthermia, multilobar chest radiograph involvement, hyponatraemia, hypo- or hyperkalaemia, low albumin, acidosis and raised C-reactive protein levels, and were more hypoxaemic.

\section{Reasons for hospitalisation of low-risk patients}

A consensus among the reviewers was achieved in 100\% of cases for assigning reasons for hospitalisation. The proportion of patients in each category is shown in figure 1 .

\section{Clinician concern}

A significant proportion of hospitalised patients (39.3\%) were classified as having high-risk clinical features requiring observation in hospital (clinician concern). Hypoxia requiring oxygen therapy was most common $(31.4 \%)$ but other common reasons were unstable comorbidities $(16.4 \%)$. These included ischaemic heart disease (acute coronary syndrome, arrhythmia and unstable angina), COPD, diabetic emergencies, severe anaemia, alcohol withdrawal and urinary retention.

$5.2 \%$ had a secondary pneumonia complication such as parapneumonic effusion or lung abscess and 5.0\% had metabolic abnormalities such as severe hyponatraemia $\left(\mathrm{Na}^{+}<130 \mathrm{mmol} \cdot \mathrm{L}^{-1}\right)$, hypo- or hyperkalaemia $\left(\mathrm{K}^{+}<3.5\right.$ or $\left.>5 \mathrm{mmol} \cdot \mathrm{L}^{-1}\right)$, or acute kidney injury requiring intravenous fluid therapy or in-patient hospital monitoring. Note that percentages are expressed as a proportion of the overall cohort of hospitalised patients $(n=420)$. Percentages sum to $>39.3 \%$ because some patients had more than one high-risk feature requiring hospitalisation.

\section{Requiring additional investigations or treatment not related to severity of pneumonia}

This group comprised $29.5 \%$ of the overall cohort and included patients that were hospitalised with none of the adverse features of severity. This group included patients requiring further investigations such as computed tomography scanning, ultrasound or bronchoscopy, where these investigations were not available rapidly as an outpatient service. $87.1 \%$ of these patients were discharged within $48 \mathrm{~h}$ of admission.

\section{Unmet social needs}

In $11.9 \%$ of cases, the reviewers could not identify any adverse clinical parameters to necessitate admission to hospital, but social circumstances were reported that would have made discharge from hospital inappropriate. These included elderly patients living alone without social support, patients whose home circumstances were unsafe or unsuitable and patients who were homeless.

\section{No clear reason for hospitalisation}

In $19.3 \%$ of cases, the reviewers could not identify a medical or social reason for the patient to be hospitalised.

\section{TABLE 1 Baseline characteristics of the study population}

\begin{tabular}{|c|c|c|c|c|}
\hline Baseline characteristics and comorbidities & Study population & \multicolumn{2}{|c|}{ CURB-65 0-1 } & p-value ${ }^{\#}$ \\
\hline Subjects $n$ & 1472 & 145 & 420 & \\
\hline Age yrs & $69(54-79)$ & $50(38-68)$ & $58(41-68)$ & 0.2 \\
\hline Males & 49.9 & 52.3 & 53.6 & 0.9 \\
\hline Liver disease & 5.1 & 3.4 & 6.7 & 0.2 \\
\hline Neurological disease & 11.8 & 3.4 & 7.1 & 0.2 \\
\hline Chronic renal failure & 6.3 & 1.4 & 1.4 & 0.9 \\
\hline Diabetes mellitus & 10.9 & 5.5 & 4.5 & 0.8 \\
\hline COPD & 22.3 & 9.0 & 21.2 & 0.02 \\
\hline Current smokers & 34.2 & 38.6 & 35.7 & 0.6 \\
\hline
\end{tabular}

Data are presented as median (interquartile range) or \%, unless otherwise stated. CURB-65: confusion, urea $>7 \mathrm{mmol} \cdot \mathrm{L}^{-1}$, respiratory frequency $\geqslant 30$ breaths $\cdot$ min $^{-1}$, systolic blood pressure $<90 \mathrm{mmHg}$ or diastolic blood pressure $\leqslant 60 \mathrm{mmHg}$, and age $\geqslant 65 \mathrm{yrs}$; COPD: chronic obstructive pulmonary disease. ${ }^{\#}$ : $\mathrm{p}$-value refers to comparison between CURB-65 0-1 groups. 
TABLE 2 Comparing parameters described in the CURB-65 scoring system between the two groups

\begin{tabular}{lccc} 
Clinical variables & Discharged from hospital in $\leqslant \mathbf{1 2} \mathbf{h} \%$ & Admitted to hospital for $>\mathbf{1 2} \mathbf{h} \%$ & $\mathbf{p}$-value \\
\hline Subjects $\mathbf{n}$ & 145 & 420 & 2.9 \\
Confusion & 0 & 15.0 & 0.08 \\
Respiratory rate $\geqslant \mathbf{3 0}$ breaths $\cdot \mathbf{m i n}^{-1}$ & 3.4 & 2.9 & 0.0004 \\
Systolic blood pressure $<\mathbf{9 0} \mathbf{~} \mathbf{m H g}$ & 2.1 & 12.1 & 0.1 \\
Diastolic blood pressure $\leqslant \mathbf{6 0} \mathbf{~} \mathbf{m H g}$ & 9.7 & 8.6 & 0.8 \\
Urea $>\mathbf{7} \mathbf{~ m m o l} \cdot \mathbf{L}^{-1}$ & 6.9 & 0.6 & \\
\hline
\end{tabular}

CURB-65: confusion, urea $>7 \mathrm{mmol} \cdot \mathrm{L}^{-1}$, respiratory frequency $\geqslant 30$ breaths $\cdot \mathrm{min}^{-1}$, systolic blood pressure $<90 \mathrm{mmHg}$ or diastolic blood pressure $\leqslant 60 \mathrm{mmHg}$, and age $\geqslant 65 \mathrm{yrs}$.

CURB-65 was documented in $17.6 \%$ of patients admitted to hospital, compared with $26.9 \%$ of patients discharged from hospital $(p=0.02)$. Of those where no clear reason could be established for hospitalisation, the CURB-65 score was documented in $17.2 \%$ of patients $(p=0.1$ when compared with patients discharged from hospital).

\section{Secondary outcomes}

Overall 30-day mortality was found to be $3.1 \%$ in those that were admitted $(>12 \mathrm{~h})$ compared with $0.7 \%$ in those that were discharged $(\leqslant 12 \mathrm{~h}) \quad(\mathrm{p}=0.1)$. The one patient who died following discharge was re-admitted and died on day 24 from a cause unrelated to CAP.

In patients classified by the reviewers as "clinical concern", mortality was $6.7 \%$. This was significantly higher compared with $0 \%$ for patients awaiting investigations $(\mathrm{p}=0.009)$ and $0 \%$ for patients without a clear indication for hospitalisation $(\mathrm{p}=0.04)$. Mortality for patients with unmet social needs was $4.0 \%$ (two patients).

In a logistic regression analysis, parameters associated with 30day mortality were chronic cardiac co-morbidity (adjusted odds ratio (aOR) 5.73, 95\% CI 1.52-21.6; $\mathrm{p}=0.01$ ); acidosis $(\mathrm{pH}<7.35)(\mathrm{aOR} 5.14,95 \% \mathrm{CI} 1.44-18.3 ; \mathrm{p}=0.01)$; hypoxia (aOR 9.86, 95\% CI 2.39-40.7; $\mathrm{p}=0.002)$; and multilobar chest radiograph shadowing (aOR 4.54, 95\% CI 1.21-17.1; $\mathrm{p}=0.03$ ). The Hosner-Lemeshow goodness-of-fit test was p-value 0.8.

Mortality increased in low-risk patients according to the number of these additional adverse features (no additional adverse features $0.3 \%$ 30-day mortality (359 patients), one adverse feature (151 patients) 2.6\%, two adverse features (45 patients) $11.1 \%$ and three adverse features (10 patients) $33.3 \%$ ). No patient had all four additional adverse features. The mortality data are shown in figure 2 .

\section{TABLE 3 Comparing various other clinical parameters between patients admitted and discharged with low CURB-65 score 0-1.}

\begin{tabular}{|c|c|c|c|}
\hline Clinical variables & Discharged from hospital in $\leqslant 12 \mathrm{~h} \%$ & Admitted to hospital for $>12 \mathrm{~h} \%$ & p-value \\
\hline Subjects $n$ & 145 & 420 & \\
\hline Temperature $<36^{\circ} \mathrm{C}$ or $>38^{\circ} \mathrm{C}$ & 24.8 & 37.9 & 0.006 \\
\hline Multilobar chest radiograph involvement & 0 & 10.5 & $<0.0001$ \\
\hline \multicolumn{4}{|l|}{ Laboratory investigations } \\
\hline Haematocrit $<30 \%$ & 1.4 & 3.1 & 0.4 \\
\hline $\mathrm{Na}^{+}<130 \mathrm{mmol} \cdot \mathrm{L}^{-1}$ & 1.4 & 6.7 & 0.04 \\
\hline $\mathrm{K}^{+}<3.5$ or $>5 \mathrm{mmol} \cdot \mathrm{L}^{-1}$ & 7.6 & 15.0 & 0.03 \\
\hline$A L T>50 \mathrm{IU} \cdot \mathrm{L}^{-1}$ & 10.8 & 8.6 & 0.6 \\
\hline$A L P>147 \mathrm{IU} \cdot \mathrm{L}^{-1}$ & 10.3 & 11.4 & 0.8 \\
\hline Albumin $<30 \mathrm{~g} \cdot \mathrm{L}^{-1}$ & 1.4 & 7.1 & 0.02 \\
\hline Arterial $\mathrm{pH}<7.35$ & 0 & 8.1 & 0.0009 \\
\hline
\end{tabular}

CURB-65: confusion, urea $>7 \mathrm{mmol} \cdot \mathrm{L}^{-1}$, respiratory frequency $\geqslant 30$ breaths $\cdot \mathrm{min}^{-1}$, systolic blood pressure $<90 \mathrm{mmHg}$ or diastolic blood pressure $\leqslant 60 \mathrm{mmHg}$, and age $\geqslant 65 \mathrm{yrs}$; ALT: alanine aminotransferase; ALP: alkaline phosphatase; CRP: C-reactive protein. ${ }^{\#}$ : defined as an arterial oxygen tension $<8 \mathrm{kPa}$ on room air, or an oxygen saturation $<92 \%$ on air in patients not undergoing arterial blood gas sampling. 


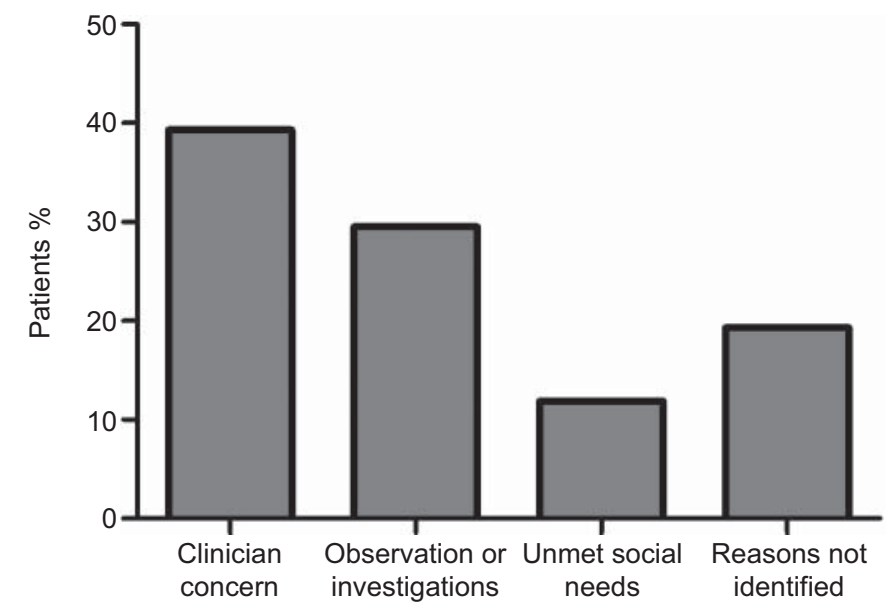

FIGURE 1. Reasons for hospital admission in patients with low CURB-65 (confusion, urea $>7 \mathrm{mmol} \cdot \mathrm{L}^{-1}$, respiratory frequency $\geqslant 30$ breaths $\cdot \mathrm{min}^{-1}$, systolic blood pressure $<90 \mathrm{mmHg}$ or diastolic blood pressure $\leqslant 60 \mathrm{mmHg}$, and age $\geqslant 65$ yrs) scores

\section{DISCUSSION}

This was the first study to investigate reasons for hospitalisation among low-risk patients with CAP in an institution using the CURB-65 score. In this study, $74.3 \%$ of the patients reviewed who had low-risk pneumonia were admitted to the hospital. Most frequently, the reasons for hospitalisation were due to additional markers of severity, such as hypoxia or unstable comorbidities. Similar proportions were not severely ill but required additional investigations. It is possible that this group of patients could have been managed as outpatients if additional resources, such as rapid access to outpatient investigations, early clinic review or "hospital at home" style support were available. We did, however, identify $19.3 \%$ of patients who independent reviewers felt could have been safely managed as outpatients. Our results are similar to a

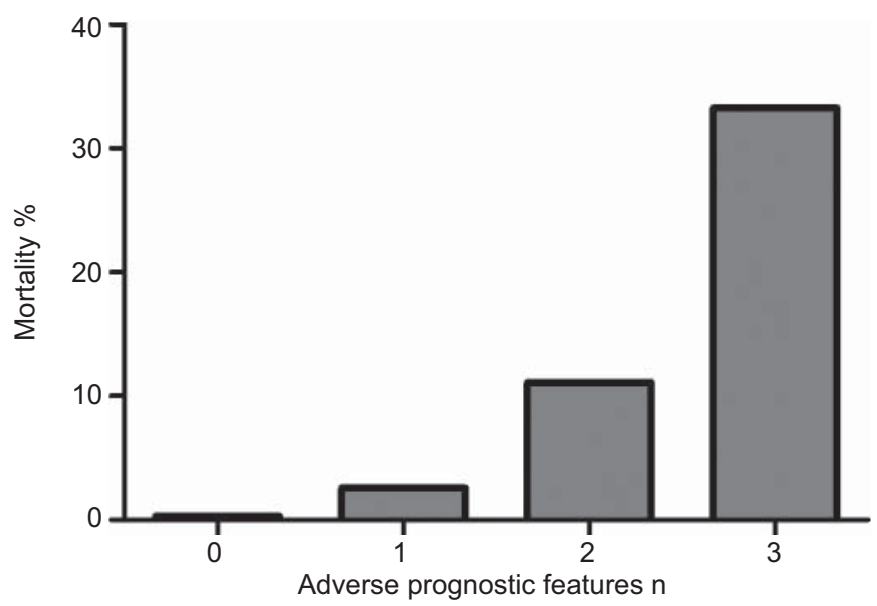

FIGURE 2. Additional severity markers and risk of mortality in patients with low CURB-65 (confusion, urea $>7 \mathrm{mmol} \cdot \mathrm{L}^{-1}$, respiratory frequency $\geqslant 30$ breaths $\cdot \mathrm{min}^{-1}$, systolic blood pressure $<90 \mathrm{mmHg}$ or diastolic blood pressure $\leqslant 60 \mathrm{mmHg}$, and age $\geqslant 65 \mathrm{yrs}$ ) scores with community-acquired pneumonia. study in the USA using the PSI, where $82 \%$ of low-risk CAP patients admitted had clinically justified reasons for hospital admission [23]. Potentially, therefore, by encouraging greater use of outpatient management and by providing additional resources for patients managed in the community, a significant number of hospital admissions for CAP could be avoided. Outpatient management of selected patients with CAP is safe, acceptable to patients and may be associated with significantly reduced hospital costs and complications [4]

In this study, where clinicians identified additional risk factors for mortality (such as hypoxia, acidosis, multilobar chest radiograph involvement or cardiac comorbidities), mortality was increased. The mortality rates for patients in whom clinicians identified additional risk factors $(6.7 \%)$ were similar to those quoted for CURB-65 score 2 in some previous studies [12]. A small proportion of patients had more than one of these severity markers and had mortality rates equivalent to those of severe CAP.

CURB-65 score is one of the most widely used pneumonia prediction scoring systems in the world. It was validated primarily to predict 30-day mortality but has recently been recommended by national guidelines to help clinicians assess the need for hospital admission [11]. Although this study is the first to investigate reasons for hospitalisation using the CURB65 score, others have studied reasons for hospitalisation of lowrisk patients using the PSI. AUJESKY et al. [16] studied 689 low-risk patients during a clinical trial that aimed to increase the proportion of patients treated in the community. In this study, the major reasons for hospitalisation were related to comorbidities $(71.5 \%)$ while patients with additional markers of severity (similar to the clinician concern category in this study) accounted for $29.3 \%$ of cases. ARNOLD et al. [23] showed that the majority of patients admitted with CAP with PSI I-II had extenuating clinical circumstances to justify their admission. Disease comorbidities (43\%) and unmet social needs $(18 \%)$ were the major categories of clinical factors justifying hospital admission for these patients. Used as the sole indicator for inappropriate hospitalisation, the PSI had a poor positive predictive value of only $16 \%$. The corresponding value for the present study is very similar (19.3\%). Hypoxia was not a major contributory factor in the study by ARNOLD et al. [23], perhaps because the PSI already incorporates oxygenation as a prognostically important factor to predict disease outcome. The study by AUJESKY et al. [16] excluded patients with arterial hypoxaemia. In addition, because age $>65$ yrs contributes 1 point to the CURB-65 score, only one more abnormal variable is required to classify an elderly patient as requiring in-patient care. This may explain why our low-risk cohort was relatively young and why previous studies consistently showed that the PSI identifies a high proportion of patients as low risk [4].

Part of the objective of this study was to study the role of clinical judgement in applying the CURB-65 score in clinical practice. Our study suggests that the CURB-65 score may be under-utilised in clinical practice, as it was not documented in most of these low-risk patients. Out of the 19.3\% patients hospitalised with no obvious justified reasons, only $17.2 \%$ had documented CURB-65 score in the notes. Had it been used, more in this group in conjunction with clinical judgement, we anticipate most of these patients could have been discharged. 
However, in most cases, clinicians appeared to appropriately identify patients with additional risk factors not included in the CURB-65 score and these patients were justifiably admitted to hospital. Where clinicians identified a cause for concern, mortality was significantly increased above the level predicted by the CURB-65 score, to a level that requires hospital treatment based on current guidelines.

This study, however, has its limitations. Although patients were prospectively recruited, we determined reasons for hospitalisation retrospectively and this approach has inherent difficulties. We accounted for potential bias by using two blinded reviewers with a third independent reviewer where consensus was not reached. A similar study using a prospective design is desirable.

\section{Conclusion}

This study supports international guideline recommendations that pneumonia severity scores should be used as an adjunct to clinical judgement when assessing the need for hospital admission. There is, however, the potential to significantly increase the proportion of patients with CAP currently managed in the community.

\section{STATEMENT OF INTEREST}

None declared.

\section{REFERENCES}

1 Trotter CL, Stuart JM, George R, et al. Increasing hospital admissions for pneumonia, England. Emerg Infect Dis 2008; 14: 727-733.

2 Armstrong GL, Conn LA, Pinner RW, et al. Trends in infectious disease mortality in the United States during the 20th century. JAMA 1999; 281: 61-66.

3 Guest JF, Morris A. Community-acquired pneumonia: the annual cost to the National Health Service in the UK. Eur Respir J 1997; 10: 1530-1534.

4 Chalmers JD, Akram AR, Hill AT. Increasing outpatient treatment of mild community-acquired pneumonia: systemic review and meta-analysis. Eur Respir J 2011; 37: 858-864.

5 Chalmers JD, Al-Khairalla M, Short PM, et al. Proposed changes to management of lower respiratory tract infections in response to the Clostridium difficile epidemic. J Antimicrob Chemother 2010; 65: 608-618.

6 Fine MJ, Auble TE, Yealy DM, et al. A prediction rule to identify low-risk patients with community-acquired pneumonia. $N$ Engl J Med 1997; 336: 243-250.

7 Lim WS, Baudouin SV, George RC, et al. BTS guidelines for the management of community acquired pneumonia in adults: update 2009. Thorax 2009; 64: Suppl. 3, iii1-iii55.

8 Mandell LA, Bartlett JG, Dowell SF, et al. Update of practice guidelines for the management of community-acquired pneumonia in imunocompetent adults. Infectious Diseases Society of America. Clin Infect Dis 2003; 37: 1405-1432.
9 Niederman MS, Mandell LA, Anzueto A, et al. Guidelines for the management of adults with community-acquired pneumonia, diagnosis, assessment of severity, antimicrobial therapy and prevention. Am J Respir Crit Care Med 2001; 163: 1730-1754.

10 Mandell LA, Marrie TJ, Grossman RF, et al. Canadian guidelines for the initial management of community-acquired pneumonia: an evidence-based update by the Canadian Infectious Diseases Society and the Canadian Thoracic Society. The Canadian Community-Acquired Pneumonia Working Group. Clin Infect Dis 2000; 31: 383-421.

11 Lim WS, Van der Erden MM, Laing R, et al. Defining communityacquired pneumonia severity on presentation to hospital: an international derivation and validation study. Thorax 2003; 58: 377-382.

12 Chalmers JD, Singanayagam A, Akram AR, et al. Severity assessment tools for predicting mortality in hospitalized patients with community acquired pneumonia. Systemic review and metaanalysis. Thorax 2010; 65: 878-883.

13 Man SY, Lee N, Ip M, et al. Prospective comparison of three predictive rules for assessing severity of community-acquired pneumonia in Hong Kong. Thorax 2007; 62: 348-353.

14 Buising KL, Thursky KA, Black JF, et al. A prospective comparison of severity scores for identifying patients with severe communityacquired pneumonia: reconsidering what is meant by severe pneumonia. Thorax 2006; 61: 419-424.

15 Aujesky D, Auble TE, Yealy DM, et al. Prospective comparison of three validated prediction rules for prognosis in community acquired pneumonia. Am J Med 2005; 118: 384-392.

16 Aujesky D, McCausland JB, Whittle J, et al. Reasons why emergency department providers do not rely on the pneumonia severity index to determine the initial site of treatment for patients with pneumonia. Clin Infect Dis 2009; 49: e100-e108.

17 Atlas SJ, Benzer TI, Borowsky LH, et al. Safely increasing the proportion of patients with community-acquired pneumonia treated as outpatients: an interventional trial. Arch Intern Med 1998; 158: 1350-1356.

18 Marrie TJ, Lau CY, Wheeler SL, et al. A controlled trial of a critical pathway for treatment of community-acquired pneumonia. CAPITAL Study Investigators. Community-Acquired Pneumonia Intervention Trial assessing levofloxacin. JAMA 2000; 283: 749-755.

19 Carratala J, Fernandez-Sabe N, Ortega L, et al. Outpatient care compared with hospitalization for community-acquired pneumonia: a randomized trial in low-risk patients. Ann Intern Med 2005; 142: 165-172.

20 Yealy DM, Auble TE, Stone RA, et al. Effect of increasing the intensity of implementing pneumonia guidelines: a randomized, controlled trial. Ann Intern Med 2005; 143: 881-894.

21 Renaud B, Coma E, Labarere J, et al. Routine use of the Pneumonia Severity Index for guiding the site-of-treatment decision of patients with pneumonia in the emergency department: a multicenter, prospective, observational, controlled cohort study. Clin Infect Dis 2007; 44: 41-49.

22 Seymann G, Barger K, Choo S, et al. Clinical judgment versus the Pneumonia Severity Index in making the admission decision. J Emerg Med 2008; 34: 261-268.

23 Arnold FW, Ramirez JA, McDonald LC, et al. Hospitalization for community-acquired pneumonia: the Pneumonia Severity Index vs clinical judgement. Chest 2003; 124: 121-124. 